\title{
Effect of in vitro aspirin stimulation on basophils in patients with aspirin-exacerbated respiratory disease
}

\author{
Gülfem E. Çelik, MD ${ }^{*}$ Professor], John T. Schroeder, PhD ${ }^{\star *}$ [Associate Professor], Robert G. \\ Hamilton, PhD ${ }^{\star *}$ [Professor], Sarbjit S. Saini, MD, PhD ${ }^{\star *}$ [Associate Professor], and N. \\ Franklin Adkinson, MD[Professor] \\ *Ankara University School of Medicine, Division of Allergy \\ ${ }^{*}$ Johns Hopkins University, School of Medicine, Asthma and Allergy Center, Division of, Allergy \\ and Clinical Immunology
}

\begin{abstract}
Background-Basophil activation has been implicated in the pathogenesis of aspirin exacerbated respiratory disease. However, a comprehensive analysis of basophil responses to aspirin in terms of mediator release, cytokine secretion and increased expression of surface activation markers has not been performed.
\end{abstract}

Objective-To study the in vitro effects of aspirin on the concurrent release of histamine, leukotriene $\mathrm{C}_{4}$ and IL-4 from human basophils and to also evaluate changes in surface activation markers (CD63, CD69 and CD203c) expressed by these cells.

Methods-Basophil-enriched cell suspensions from 10 patients with aspirin exacerbated respiratory disease and 10 healthy volunteers were incubated with lysine-aspirin for up to 3 hours. Cells were analysed for expression of CD63, CD69 and CD203c using flow cytometry. Cell-free supernatants were evaluated for histamine, and leukotriene $\mathrm{C}_{4}$ release and for IL-4 secretion.

Results-Aspirin-induced expression of CD63, CD69 and CD203c yielded 30\%,80\% and 70\% sensitivity, respectively, but with poor specificity. There was no significant difference in leukotriene $\mathrm{C}_{4}$ synthesis between groups. None of the patients with aspirin exacerbated respiratory disease (or controls) released IL-4 in response to aspirin. A higher dose of $5 \mathrm{mg} / \mathrm{ml}$ aspirin mediated non-specific effects on basophils.

Conclusion-Basophil responses to in vitro aspirin challenge are poor indicators of clinical sensitivity. Aspirin activates some basophils by means of mechanisms which differ from the classical IgE mediated pathway. Our study also shows that the use of $27 \mathrm{mM}$ of aspirin $(5 \mathrm{mg} / \mathrm{ml})$ used by previous investigators causes nonspecific basophil activation, thereby eliminating its usefulness in a cell based diagnostic test for AERD. Evaluation of in vitro basophil activation has low clinical value in identifying aspirin- induced respiratory reactions

\section{Keywords}

Aspirin; asthma; aspirin exacerbated respiratory disease; basophil; CD63; CD69; CD203c; histamine; leukotriene $\mathrm{C}_{4}$; flow cytometry

Adress for correspondence: Gülfem E. Çelik, Ema Asma Bahce Bloklari C-9, Cayyolu-Yenimahalle, 06580, Ankara-Turkey, Tel: +90 312 5957155, Fax: +90 312 3190046, Email: gulfemcelik@gmail.com. 


\section{INTRODUCTION}

Aspirin exacerbated respiratory disease (AERD) is a clinical phenotype of asthma in which aspirin (ASA) and other nonsteroidal anti-inflammatory drugs (NSAIDs) cause asthma flares and rhinoconjunctivitis [1,2]. Although many attempts have been made to understand the mechanisms leading to AERD, they have remained elusive. Arachidonic acid (AA) pathway metabolites or enzymes have always been a focus of studies on the pathogenesis of AERD [3-5]. Both therapeutic and adverse effects of aspirin and other NSAIDs are believed to be caused by inhibition of cyclooxygenase (COX), an enzyme which is responsible for synthesis of prostaglandins (PG) from AA [3-5]. Over the last years, cysteinyl leukotrienes (Cys-LTs) namely, leukotriene (LT) $\mathrm{C}_{4}, \mathrm{LTD}_{4}$ and $\mathrm{LTE}_{4}$ have emerged as major mediators in the pathogenesis of AERD [6-10]. Decreased levels of $\mathrm{PGE}_{2}$, and increased levels of $\mathrm{PGD}_{2}$ and 15-hydroxyeicosatetraenoic acid (HETE) are among the other mechanisms suggested for the pathogenesis of AERD [11-13].

Recent studies have shown evidence that basophils are involved in AERD. In vivo studies have indicated a higher $\mathrm{LTC}_{4}$ release in AERD patients, which makes the basophil, and eosinophil potential sources for this release [7,8]. In addition, studies evaluating the surface expression of CD63 by flow cytometry have indicated a role for basophils and even recommended the use of CD63 expression as a diagnostic marker of the disease [14,15]. As a member of the transmembrane-4 superfamily, CD63 is rapidly upregulated after IgE/ receptor cross-linking by allergen[16,17]. Several groups have used CD63 expression in the diagnosis of IgE-mediated allergy, including those linked to latex, venom, pollen and foodassociated allergies[18-19. However, the sensitivity and specificity of these assays and their validation require independent confirmation. Determination of CD63 expression also has been shown to occur with reactions to certain drugs such as neuromuscular agents and metamizole in which IgE-mediated reactions are implicated [22-25].

Other surface activation markers on basophils have also been implicated has having utility in detecting IgE-dependent activation. In particular, CD203c [ectonucleotide pyrophosphatase (E-NPP3)], has been reported to be a superior marker to CD63[26,27]. Unlike CD63, its expression is unique to basophils, mast cells and their progenitors [16,17]. CD69 is another surface marker that is increased on basophils following IgE-mediated activation [28].

However, since the expression of this molecule is also increased following IL-3 exposure, its use is limited in determining IgE-dependent reactions. Moreover, CD69 is expressed by several different cell types including eosinophils, and T cells, [29,30] and is thus not specific to basophils.

Basophils respond to IgE-dependent stimulation by not only expressing increased levels of activation markers or by releasing histamine and $\mathrm{LTC}_{4}$, but also by secreting large quantities of IL-4 and IL-13 [16,17]. However, it is not known whether basophils react to aspirin in patients with AERD by secreting greater levels of these cytokines or any other mediator. In this study, we evaluate basophil responses to aspirin by attempting to confirm reports that this drug selectively induces the expression of the activation markers (i,e. CD63). We also aim to study other basophil surface markers of CD69 and CD203c in AERD subjects and further evaluate whether histamine, IL- 4 and $\mathrm{LTC}_{4}$ are induced by aspirin in patients compared to healthy controls.

\section{MATERIAL AND METHODS}

\section{Study Subjects}

The study population consisted of two different groups: Group 1: Ten patients with AERD as diagnosed by clinically documented asthmatic responses after ingestion of ASA or other 
NSAIDs; Group 2: Ten healthy volunteers without asthma or analgesic intolerance as controls. Asthma diagnosis was based on a history of recurrent symptoms of wheezing, shortness of breath, cough and demonstration of objective signs of reversible airway obstruction by means of at least $>12 \%$ increase in $\mathrm{FEV}_{1}$ after 15 minutes with an inhalation of $200 \mu \mathrm{g}$ salbutamol according to guidelines [31]. None of the asthmatic patients were on specific immunotherapy. Subjects with certain and reliable histories of aspirin sensitivity (two separate episodes of severe asthma after use of aspirin or NSAID requiring emergency room visits and documented by a physician within the last 5 years), and patients with recent positive oral aspirin provocation tests were both included in the study. Control subjects (reported safe intake of aspirin or other NSAIDs within the last month) were also recruited for study. Subjects who had taken any NSAIDs/ASA or oral antihistamines within the last 5 days were excluded from the study. This study was approved by the local institutional review board and each subject provided written informed consent prior to the participation.

\section{Basophil Studies}

Reagents used for experiment-Piperazine-N, N0-bis-2-ethanesulphonic acid (PIPES) (Sigma Chemical Co., St Louis, MO, USA) buffer 10X (250 mM/L PIPES, $1.10 \mathrm{M} \mathrm{NaCl}, 50$ $\mathrm{mM} \mathrm{KCl}, \mathrm{pH} 7.3$ ) was stored at $4{ }^{\circ} \mathrm{C}$ as a stock solution and used for preparation of $1 \mathrm{X}$ PIPES and PAG/PAG-EDTA buffers.

1X PIPES was prepared by 1:10 dilution of 10X PIPES. Isotonic Percoll (100\% Percoll) (Pharmacia Biotec, Inc, Piscataway, NJ, USA) was prepared by mixing 1 part $10 \times$ PIPES and 9 parts Percoll. Working solutions of Percoll at 55\% (density, $1.072 \mathrm{~g} / \mathrm{mL}$ ) and $61 \%$ (density, $1.081 \mathrm{~g} / \mathrm{mL}$ ) were made by mixing the appropriate amounts of $100 \%$ Percoll with $1 \times$ PIPES.

Conditioned medium (C-IMDM) consisted of IMDM supplemented with $1 \times$ non-essential amino acids, and $5 \mu \mathrm{g} / \mathrm{mL}$ gentamicin (all 3 from Life Technologies, Inc, Grand Island, NY, USA) and $5 \%$ heat-inactivated $\left(56^{\circ} \mathrm{C}\right.$ for 30 minutes) fetal bovine serum (FBS) (Sigma Chemical Co. St Louis, MO, USA).

PIPES/albumin/glucose (PAG) contained one-tenth 10X PIPES, 0.003\% HSA and 0.1\% dglucose. PAG-EDTA additionally contained $4 \mathrm{mM}$ EDTA.

Lysine-aspirin (Ivy chemicals, NJ, USA) was weighted and stored at $+4{ }^{\circ} \mathrm{C}$ and diluted freshly with $1 X$ PIPES.

Bovine serum albumin (BSA) dissolved in a phosphate buffer saline (PBS) in $0.1 \%$ rate and $10 \%$ DMSO contained 1 part DMSO and 9 part PBS were used during preparing the cells for flow cytometry.

Protocol: Sixty ml of venous blood was freshly drawn into polypropylene syringes containing $5 \mathrm{ml}$ of $0.1 \mathrm{M}$ EDTA to prevent coagulation. Enriched basophils were obtained by density-gradient sedimentation, using a previously described double Percoll gradient procedure [32]. Buffy-coat cell suspensions (prepared by centrifuging the anti-coagulated blood at 300XG for $15 \mathrm{~min}$.) were diluted 1:1 v/v with PAG-EDTA buffer and carefully layered onto the Percoll gradients. After centrifugation at 700Xg for 20 min., - containing 1X PIPES, $0.003 \%$ HSA, $0.1 \%$ d-glucose and $4 \mathrm{mM}$ EDTA in order to reduce platelet aggregation during their removal using low-speed (100xg) centrifugation-, basophils were quantified by counting Alcian blue positive stained cells on a Spiers-Levy chamber.

For each condition, $0.125 \mathrm{ml}$ of $2 \mathrm{X}$ ( 2 times the final concentration) of stimulus in C-IMDM in duplicates were placed in $1.5 \mathrm{ml}$ RNA-se free polyproplene microcentrifuge tubes. Doses 
of aspirin included those previously used by Sanz et al.[14] which included 5, 1.25 and 0.3 $\mathrm{mg} / \mathrm{ml}$ as well as inclusion of additional pharmacologic doses. Therefore, final aspirin concentrations for this experiment were $0.001,0.01,0.1,1.6$ (equivalent to $0.3 \mathrm{mg} / \mathrm{ml}$ ), 6.9 (equivalent to $1.25 \mathrm{mg} / \mathrm{ml}$ ) and $27 \mathrm{mM}$ (equivalent to $5 \mathrm{mg} / \mathrm{ml}$ ). A goat anti-human $\mathrm{IgE}$ (final concentration: $20 \mathrm{ng} / \mathrm{ml}$ ) and A23187 (final concentration: $100 \mathrm{nM}$ ) (Sigma Chemical Co. St Louis, MO, USA) were used as positive control stimuli. C-IMDM alone served as a negative control to evaluate background stimulation.

The basophil enriched leukocyte suspension was suspended in C-IMDM and both stimuli and cells were pre-warmed to $37^{\circ} \mathrm{C}$ for 15 minutes which was followed by addition of 0.125 $\mathrm{ml}$ of cell suspension to the tubes containing stimulus.

Analytic measurements-In this study, all of the experimental variables were optimized using the same culture environment. As IL-4 synthesis requires a 3 hour incubation, we performed a preliminary experiment for time-kinetics of expression markers of CD63, CD69 and CD203c with an anti-IgE stimulus and showed that expression of these markers were still high at 3 hours culture period Therefore, after stimulation, cell cultures were subsequently incubated at $37^{\circ} \mathrm{C}$ in a $5 \% \mathrm{CO}_{2}$ incubator $\left(\mathrm{CO}_{2}\right.$ water jacketed incubator Forma Scientific, USA) for 3 hours (34). For harvesting, cultures were centrifuged and cellfree supernatants were collected for histamine, and $\mathrm{LTC}_{4}$ and IL-4 quantification.

Flow cytometry Studies-In our preliminary experiment, we investigated the effect of different staining techniques of cells for flow cytometry on basophil surface marker expression. In a parallel design study, we compared two methods for flow cytometry analysis in terms of staining of previously fixed cells and fresh staining followed by fixing procedure. We obtained comparable results with both methods Thus, a fixing procedure was used in all subsequent experiments [33] basophil-enriched cell cultures were harvested, duplicate cultures were pooled and cells were washed with PBS. The cell pellets were then fixed by addition of $0.3 \mathrm{ml}$ of $4 \%$ buffered paraformaldehyde, followed by an additional wash and storage at $-70^{\circ} \mathrm{C}$ in $0.300 \mathrm{ml}$ of PBS- $10 \%$ DMSO until analysis.

Cell staining for flow cytometry was performed using the following antibodies: 1) APCconjugated mouse IgG2b as an isotype control (eBiosciences, San Diego, CA USA); 2) antihuman Anti-FceR1 $\alpha$ (eBiosciences); 3, phycoerythrin (PE)-conjugated mouse IgG1 (BD Pharminogen, San Jose, CA, USA); 4, PE-conjugated anti-human CD63 (BD Pharminogen); 5, PE-conjugated anti-human CD69 (BD Pharminogen,), and 6, PE-conjugated anti-human CD203c (Immunotech, Marseille, France). Cells were first incubated with a blocking solution containing PBS with 5\% nonfat milk and $1 \mathrm{mg} / \mathrm{ml}$ human IgG to block nonspecific binding to FcR. After incubating $1 \mathrm{~h}$ on ice, the cell suspension was distributed among fresh $1.5 \mathrm{ml}$ microcentrifuge tubes in $0.025 \mathrm{ml}$ aliquots. An equal amount of different antibody cocktails (at $2 \mathrm{X}$ the final concentration) was then added to appropriate tubes for $30 \mathrm{~min}$. incubation on ice and covered with aluminum foil. The cells were then washed $3 \mathrm{X}$ with PBS before resuspending in $0.300 \mathrm{ml}$ PBS $0.1 \%$ BSA.

Samples were analysed on a Becton Dickinson (Franklin Lakes, NJ, USA) FACS Caliber flow cytometer employing Cell Quest software (BD Biosciences, Franklin Lakes, NJ, USA). Cells were initially gated based on APC-FceR $1 \alpha$-positive cells to select the basophil population and then analysed for specific activation markers (Figure 1). At least 500 basophils were analysed. The data are expressed as net mean fluorescence intensity (MFI) (actual MFI-MFI of irrelevant IgG control) and stimulation index (SI) (stimulated/ unstimulated MFI). A SI >2 was considered positive. 
Histamine releasing Activity: Cell-free supernatant $(0.05 \mathrm{ml})$, carefully removed from BEC culture following incubation, was diluted in $1 \mathrm{~mL}$ of PAG buffer containing $8 \%$ $\mathrm{HClO}_{4}$, acid-precipitated overnight at $+4^{\circ} \mathrm{C}$, and analysed for histamine via automated fluorimetry. Results are shown as the percentage of total histamine content after subtracting spontaneous release and negative control.

Leukotriene $\mathbf{C}_{4}$ : Leukotriene $\mathrm{C}_{4}$ release was measured with a competitive enzyme immunoassay (Cayman Chemicals, Ann Arbor, USA). In the measurement, $\mathrm{LTC}_{4}$ antiserum, $\mathrm{LTC}_{4}$ acetylcholine esterase tracer, and standard for $\mathrm{LTC}_{4}$ were used. Adsorbance was measured at $415 \mathrm{~nm}$. A standard curve was run in each plate using 8 concentrations that ranged from 7.8 to $1000 \mathrm{pg} / \mathrm{ml}$. The $\mathrm{LTC}_{4}$ concentration was interpolated using a four parameter logistic equation. Values were normalized to $10^{6}$ basophils.

IL-4 measurements: IL-4 levels in the basophil culture supernatants were measured with an enzyme-linked immunosorbent assay (eBiosciences). The standard curve ranged from 2 to $200 \mathrm{pg} / \mathrm{ml}$. Values were normalized to $10^{6}$ basophils.

Statistical analysis: Data analysis was performed with SPSS (Statistical Package for Social Sciences v11.0, Chicago, IL, USA). Numeric parameters are expressed as mean \pm SEM. Ordinal values are given as $n(\%)$. Nonparametric tests were used for comparative analysis. Inter-group comparisons were performed by Kruskal Wallis $(K W)$ analysis and in the case of significant $\mathrm{p}$ values after KW test, a posthoc Mann-Whitney U test was used. A Chi-square test was used for comparison of ordinal and nominal values. Correlation analyses were performed by a Pearson correlation test. A $p$ value less then 0.05 was considered significant.

\section{Results}

\section{Patient Demographics}

The study group included 10 patients with AERD (Female/male: 4/6, mean age: $55 \pm 3.5$ years old) [Table 1]. and 10 control subjects (Female/male:7/3, mean age:45 \pm 3 years old) Six out of 10 AERD patients(60\%) had positive skin prick test results. Except for one case who required oral steroids, all asthmatic subjects were using a combination of medicines that included inhaled steroids and a long acting beta2 agonist. In these combination medicines the doses of fluticasone were $200 \mathrm{mcg}$ daily in 2 cases, $500 \mathrm{mcg}$ daily in 4 cases and $1000 \mathrm{mcg}$ daily in 4 cases. The one subject using oral steroids stopped the medication 2 days prior to blood collection. No AERD subject was using oral anti-leukotriene drugs.

\section{Basophil Studies}

CD63-The baseline CD63 expression in basophils from both groups was comparable (AERD: $246 \pm 26$ MFI vs controls:270 \pm 27 MFI, Figure 2). Stimulation with anti-IgE markedly induced CD63 expression, but this was not significantly different between the two

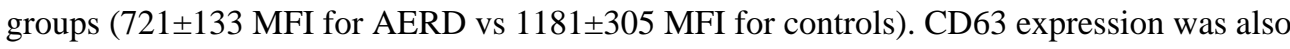
equally induced on basophils from both groups when using the calcium ionophore, A23187 (AERD: 2958 \pm 227 MFI vs Controls: 2504 \pm 194 MFI). In fact, the levels of CD63 induced by A23187 were 3- to 5-fold greater than those seen with anti-IgE. While aspirin-stimulated specimens showed a significant increase in CD63 expression compared to cells cultured in medium alone, the effect was observed equally in AERD patients and normal subjects.. The SI was $>2$ in 3 patients with AERD (30\%) and in 6 control subjects (60\%). Specificity of CD63 for detecting aspirin induced reactions was $40 \%$. 
CD203c - Baseline CD203c expression was similar for the two groups (AERD: 106 \pm 13 MFI vs controls:76 \pm 6.5 MFI Figure 3). Activation with positive control stimuli increased the levels of CD203c on basophils equally for patients and controls (anti-IgE stimulation: $321 \pm 49$ MFI for AERD vs $288 \pm 49$ MFI for controls and A23187 stimulation: AERD: $535 \pm 57.3$ MFI vs controls:445 \pm 41 MFI, Figure 3).

Although aspirin stimulation produced minimal changes in CD203c expression in both groups, there was a quite evident induction of CD203c at the highest dose of aspirin tested (i.e. $27 \mathrm{mM}$ ), which was significantly different from all other aspirin doses $(\mathrm{p}=0.001$ for AERD and $\mathrm{p}=0.0006$ for controls, Figure 3 ). There was no difference between the two groups when comparing all corresponding doses of aspirin. There was linear trends for aspirin's effects on CD203c expression among the two groups of subjects. SI was over 2 in 7 out of 10 patients (70\%) with AERD and 5 out of $9(55 \%)$ control subjects being mostly positive at the highest concentration of aspirin $(27 \mathrm{mM})$. However, the specificity of $\mathrm{CD} 203 \mathrm{c}$ for determining aspirin induced reactions was $45 \%$.

CD69-CD69 expression on basophils was more than 10-fold lower than other activation markers investigated. CD69 levels on basophils cultured in medium alone were 5.5 $\pm 1.4 \mathrm{MFI}$ for AERD vs. $9 \pm 3.9$ MFI for controls, and did not significantly differ between groups. As shown in Figure 4, anti-IgE stimulation increased this expression approximately 7 -fold for both groups (AERD: $45.4 \pm 11.9$ MFI vs controls: 67.3 \pm 17.1 MFI, p>0.05), which was similarly induced when using A23187 (AERD: 78 \pm 10.9 MFI vs Controls:82 \pm 8.1 MFI, Figure 4).

Aspirin produced modest but equivalent increases in CD69 on basophils from both the patient and normal control group. The stimulated specimens showed a significant increase in both groups. There was no difference in CD69 expressions within groups with any of the aspirin doses compared to buffer control.

When the stimulation index curves were plotted for CD69, similar levels of CD203c were observed using the highest concentration of aspirin $(27 \mathrm{mM})$ ). The stimulation index was $>2$ in 8 out of $10(80 \%)$ patients and 6 out of $9(66 \%)$ control subjects. Specificity of CD69 was $34 \%$.

$\mathrm{LTC}_{4}$ release-There was no detectable $\mathrm{LTC}_{4}$ levels in medium alone conditions. Both anti-IgE and A23187 stimuli induced the release of comparable levels of $\mathrm{LTC}_{4}$ in AERD patients and normal subjects (Anti-IgE $=155 \pm 55.7 \mathrm{pg} / 10^{6}$ cells in AERD and $136 \mathrm{pg} / 10^{6}$ cells in controls, A23187 $=2101 \pm 353 \mathrm{pg} / 10^{6}$ cells in AERD vs $2587 \pm 81 \mathrm{pg} / 10^{6}$ cells in controls) (Figure 5).

Although corresponding doses of aspirin induced similar results in each group (Figure 5), the percentage of cases with detectable $\mathrm{LTC}_{4}$ levels at 0.001 and $0.1 \mathrm{mM}$ of aspirin were higher in the AERD group as compared to the control group (aspirin $0.001 \mathrm{mM}: 40 \%$ vs $20 \%$, $\mathrm{p}=0.01$, aspirin $0.01 \mathrm{mM}$ : $80 \%$ vs $30 \%$, $\mathrm{p}=0.001$, aspirin $0.1 \mathrm{mM}: 70 \%$ vs $30 \%$, $\mathrm{p}=0.001$ ). The peak level for $\mathrm{LTC}_{4}$ release was seen at $27 \mathrm{mM}$ dose of aspirin in each group and these levels were comparable $\left(\mathrm{AERD}=310 \pm 42 \mathrm{pg} / 10^{6}\right.$ cells vs patients $=253 \pm 24 \mathrm{pg} / 10^{6}$ cells).

Histamine release-In this study, histamine-releasing activity was defined as $5 \%$ histamine release above the spontaneous release values, which were typically $<5 \%$ of total histamine. Using this criterion, aspirin induced histamine release in 6 out of 10 subjects (60\%) with AERD and 2 out of 9 controls (22\%). However, histamine release was evident only at the $27 \mathrm{mM}$ concentration. For release to anti-IgE, a suboptimal concentration ( $20 \mathrm{ng} /$ 
$\mathrm{ml}$ ) was used, since this dose was better at inducing IL-4 (see below). Therefore, only six subjects in each group had over 5\% histamine release to anti-IgE stimuli. Three patients with AERD and 2 control subjects had a release greater than $20 \%$ of total histamine content to anti-IgE stimuli. Histamine was also released from all subjects with A23187 (AERD:132 \pm 42 vs controls: $81 \pm 15$ )

IL-4 release-None of the patients with AERD released IL-4 with stimulation to any doses of aspirin (data not shown). However, one control subject had detectable levels of IL-4 that was detected with all doses of aspirin as well as medium alone. Other control subjects did not show any IL-4 release with aspirin. IL-4 induced with anti-IgE stimulation was generally less among subjects with AERD, but this was not significantly different $\left(46 \pm 15.6 \mathrm{pg} / 10^{6}\right.$ cells among AERD patients vs. $132 \pm 66 \mathrm{pg} / 10^{6}$ cells in control subjects; $\mathrm{p}>0.05$ ). A23187 markedly induced IL-4 in both groups of subjects (AERD:1069 $\pm 461 \mathrm{pg} / 10^{6}$ vs controls: $936 \pm 242 \mathrm{pg} / 10^{6} \mathrm{p}>0.05$ )

\section{Correlations between aspirin- vs. anti-lgE- dependent induction of activation} markers-While aspirin showed an inability to selectively induce surface activation markers described above in AERD patients vs. normal controls, several unexpected correlations were seen when comparing activation markers induced by these stimuli. Although there were correlations between several doses of aspirin and anti-IgE response in CD63 expressions, the most significant correlations were observed between the low doses of aspirin and anti-IgE response $(r=0.699, p=0.002)$. For CD203c expressions, only significant correlations were observed between the expression of these markers at the two highest concentrations ie $27 \mathrm{mM}$ and $6.9 \mathrm{mM}$ of aspirin and in response to anti- $\operatorname{IgE}$ stimulation regardless of the underlying aspirin sensitivity $(\mathrm{r}=0.581, \mathrm{p}=0.0012)$. A similar pattern was also evident for CD69, but the most significant correlation was seen at $27 \mathrm{mM}$ of aspirin and anti-IgE stimulation $(r=0.625, p=0.007, .$. There was no correlation observed for $\mathrm{LTC}_{4}$ release to aspirin and anti-IgE.

\section{Discussion}

In this study, we examined basophil responses to aspirin in terms of IL-4 production, $\mathrm{LTC}_{4}$ and histamine release and the expression of basophil surface activation markers (CD63, CD69 and CD203c). Responses were studied to several doses of aspirin including both pharmacological doses for COX-1 enzyme inhibition and those higher doses employed in previous in vitro studies of flow cytometry in patients with AERD and controls. We employed a dose-response study in an attempt to identify a dose related effect of aspirin on basophil function. Our data indicate that high concentrations of aspirin produce non-specific activation of basophils. Overall, these results indicate that the quantization of basophil activation markers has a low sensitivity and specificity for identifying patients with AERD.

Finding a safe and reliable diagnostic test as an alternative to aspirin challenge for diagnosis of AERD has always been an important goal. In recent studies [14,15], aspirin induced CD63 expression in patients with AERD and/or urticaria showed sensitivity and specificity rates of $40 \%$ and $100 \%$, respectively, with a single analgesic. The diagnostic sensitivity increased to $67 \%$ specificity with the use of 4 different analgesics. In contrast, our study found that aspirin induced expression of CD63 had low sensitivity (30\%) for diagnosis of AERD.

This negative finding could be explained by differences in the methodology and doses of aspirin used in our study compared to those utilized by other investigators. In particular, Sanz et al. used high, supra-pharmacologic concentrations of aspirin [5 mg/ml, $(27 \mathrm{mM})$, $1.25 \mathrm{mg} / \mathrm{ml}(6.9 \mathrm{mM})$ and $0.3 \mathrm{mg} / \mathrm{ml}(1.6 \mathrm{mM})]$ [14]. In our study, the most impressive data 
was obtained with an aspirin dose of $27 \mathrm{mM}$. Regardless of any underlying aspirin sensitivity, this highest dose of aspirin caused non-specific basophil activation as measured by the expression, of CD69 and CD203c, as well as $\mathrm{LTC}_{4}$ and histamine release. Our data indicate that high concentrations of aspirin produce non-specific activation of basophils. Among patients undergoing oral ASA challenge, simultaneous determination of serum aspirin level was found to be between 2.9 and 33.3 micromol when the aspirin provocation test was positive [34] We, thus, chose to discount results with $5 \mathrm{mg} / \mathrm{ml}(27 \mathrm{mM})$ in our study, which decreased the diagnostic sensitivity further to $20 \%$.

On the other hand, our methodology also differed from previous studies. We used basophil enriched preparations obtained by double Percoll gradients, rather than dextran-sedimented leukocyte preparations used in previous studies. In this method, the percentage of basophils in these suspensions typically range between 5-20\%, depending on the subject and the percentage of basophils between the two groups did not significantly differ. Although a few DCs (primarily pDCs) are still found in the basophil enriched cell (BEC) suspensions, they typically account for less than $0.15 \%$, which is some 33 -fold less than even our most impure basophil frequencies. Overall, the DC contribution was negligible at best.. Moreover, in our method, the cells were initially gated based on APC- FcER $1 \alpha$-positive cells to select the basophil population and then analysed for specific activation markers. By selection of these cells, platelets and any dendritic cells were gated out. So, this enrichment step eliminated cells such as platelets, eosinophils and dendritic cells that are known to confound surface expression and mediator release studies [16,17]. It also allowed us to concurrently investigate basophil mediators and surface markers from the same culture. The use of whole blood and dextran sedimented leukocytes for assessment of flow cytometry may have led to false positive results in previous studies. Increased expression of CD63 seen n some previous studies could have been mediated byplatelets and dendritic cells, both of which respond to NSAIDs [14,15].

On the other hand, differences in methodology between these earlier studies and ours limit direct comparison of the results. Moreover, in a recent article, Malbran and colleagues also showed that the NSAID diclofenac did not induce CD63 expression in basophils of patients with diclofenac sensitivity [35]. Taken together these results support the lack of usefulness of evaluating basophil CD63 expression by flow cytometry in the diagnosis of AERD.

Although encouraging results have been published that indicate the expression of CD203c is a useful marker of basophil activation for IgE mediated reactions, we are not aware of any study that has used this marker in the diagnosis of AERD. Our results provide evidence that $\mathrm{CD} 203 \mathrm{c}$ is no better than CD63, in the evaluation of AERD. Additionally, CD69 produced a diagnostic sensitivity and specificity in our study of 50\% and 66\%, respectively, even when excluding the highest nonspecific triggering dose of aspirin.

In general, the basophil surface markers all had a low diagnostic specificity for detecting aspirin-induced reactions. Aspirin activates basophils, even from non-aspirin sensitive control subjects. The strong correlations between surface marker expression induced by aspirin and anti-IgE could result from some common intracellular pathways, which have no relationship to the pathogenesis of AERD.

Increased CysLT production has been documented in patients with AERD [3-10]. In vivo studies in which human secretions such as bronchoalveolar lavage fluid (BAL) and urine were collected, provide strong evidence for Cys LT enhancement and possible involvement in this disease [3-5,7, 89. However, in vitro studies have shown conflicting results [6,9-10]. Our study found that despite a trend for higher levels of Cys LT in patients with AERD, patients could not be distinguished from normal controls by in vitro challenge with a wide 
range of doses of aspirin. Similar to the data obtained with flow cytometry, the maximum $\mathrm{LTC}_{4}$ release in both groups was seen at the highest dose of aspirin $(27 \mathrm{mM})$ in all cases. These data support the conclusion of a nonspecific basophil activation at higher aspirin doses and they parallel our findings obtained by flow cytometry. An aspirin dose between $0.001 \mathrm{mM}$ and $0.1 \mathrm{mM}$ was, however, more discriminating of AERD as it was negative in many control subjects.

Previous studies have showed the limited utility of basophil histamine release in response to aspirin as a marker for AERD [36,37]. Excluding the toxic responses obtained at the highest dose of aspirin, our results confirm the low diagnostic sensitivity and specificity of basophil histamine release.

As far as we know, IL-4 release with aspirin stimulation has not been studied previously. Although IL-4 is released from basophils after crosslinking with anti-IgE, [16,17,38-40] it was of interest to determine whether any IL-4 would be released from basophils after incubation with aspirin in vitro. Our results clearly show that aspirin-induced basophil activiation, whether with cells from AERD patients or normal controls, did not consistently initiate IL4 production.

In conclusion, in this study, we showed that the use of $27 \mathrm{mM}$ of aspirin $(5 \mathrm{mg} / \mathrm{ml})$ used by previous investigators causes nonspecific basophil activation, thereby eliminating its usefulness in a cell based diagnostic test for AERD. We also observed that $\mathrm{LTC}_{4}$ release from basophils collected from patients with AERD is not correlated with histamine and IL-4 release or increased expression of basophil surface activation markers. These data indicate that aspirin can activate some basophils by mechanism(s) which differ from IgE mediated pathways. On the other hand, although our methodology differed from previous publications some of which have found increased expression of CD63 in patients with AERD, other methological differences make difficult the direct comparison of results. We also showed that at lower concentrations of aspirin, it was not possible to distinguish AERD patients from normal controls using any of 3 basophil activation markers, or histamine or leukotriene release. Basophil activation surface marker expression appears to be influenced by intrinsic histamine releasibilityby allergen or anti-IgE regardless of a comorbid aspirin sensitivity. Future studies will need to focus on other basophil markers or processes, and perhaps examine other cell types which may be involved in the pathogenesis of AERD.

\section{Acknowledgments}

Supported in part by NIH Grants AI143654F (NF. Adkinson), and RO1A142221 (John T. Schroereder). Gulfem E. Celik was supported by TUBITAK and TUBA-GEBIP. The authors would like to thank to Anja Bieneman, Khristin Chicester and Sherry Hudson for their technical supports and to Victoria Santapietro and Deborah Bull for their help in blood drawing.

\section{Abbrevation list (in alphabetic order)}

AA

AERD

ASA

ATA

COX

CysLT

ELISA
Arachidonic acid

Aspirin Exacerbated Respiratory Disease

Aspirin

Aspirin Tolerant Asthmatics

Cyclooxygenase

Cysteinyl leukotriene

Enzyme linked immunoSorbent assay 


$\begin{array}{ll}\text { E-NPP3 } & \text { ectonucleotide pyrophosphatase } \\ \text { HETE } & \text { Hydroxyeicosatetraenoic acid } \\ \text { LT } & \text { Leukotriene } \\ \text { NSAID } & \text { Nonsteroidal anti-inflammatory drug } \\ \text { PG } & \text { Prostaglandin } \\ \text { PIPES } & \text { Piperazine-N, N0-bis-2-ethanesulphonic acid }\end{array}$

\section{References}

1. Stevenson DD, Szczeklik A. Clinical and pathological perspective on aspirin sensitivity and asthma. J Allergy Clin Immunol. 2006; 118:773-86. [PubMed: 17030227]

2. Kowalski ML. Aspirin-sensitive rhinosinusitis and asthma. Clin Allergy Immunol. 2007; 19:147-75. [PubMed: 17153012]

3. Szczeklik A, Sanak M. The broken balance in aspirin hypersensitivity. Eur J Pharmacol. 2006; 533:145-55. [PubMed: 16457808]

4. Szczeklik A, Sanak M, Nizankowska-Mogilnicka E, Kielbasa B. Aspirin intolerance and the cyclooxygenase-leukotriene pathways. Curr Opin Pulm Med. 2004; 10:51-6. [PubMed: 14749606]

5. Farooque S, Lee T. Mechanisms of aspirin-sensitive respiratory disease--a two-compartment model. Int Arch Allergy Immunol. 2007; 142:59-63. [PubMed: 17016058]

6. Celik G, Bavbek S, Misirligil Z, Melli M. Release of cysteinyl leukotrienes with aspirin stimulation and effect of prostaglandin E2 on this release. Clin Exp Allergy. 2001; 31:1615-22. [PubMed: 11678863]

7. Higashi N, Taniguchi M, Mita H, Kawagishi Y, Ishii T, Higashi A, et al. Clinical features of asthmatic patients with increased urinary leukotriene E4 excretion (hyperleukotrienuria): Involvement of chronic hyperplastic rhinosinusitis with nasal polyposis. J Allergy Clin Immunol. 2004; 113:277-83. [PubMed: 14767442]

8. Antczak A, Montuschi P, Kharitonov S, Gorski P, Barnes PJ. Increased exhaled cysteinylleukotrienes and 8-isoprostane in aspirin-induced asthma. Am J Respir Crit Care Med. 2002; 166:301-6. [PubMed: 12153961]

9. Pierzchalska M, Mastalerz L, Sanak M, Zazula M, Szczeklik A. A moderate and unspecific release of cysteinyl leukotrienes by aspirin from peripheral blood leucocytes precludes its value for aspirin sensitivity testing in asthma. Clin Exp Allergy. 2000; 30:1785-91. [PubMed: 11122218]

10. May A, Weber A, Gall H, Kaufmann R, Zollner TM. Means of increasing sensitivity of an in vitro diagnostic test for aspirin intolerance. Clin Exp Allergy. 1999; 29:1402-11. [PubMed: 10520062]

11. Pierzchalska M, Szabo Z, Sanak M, Soja J, Szczeklik A. Deficient prostaglandin E2 production by bronchial fibroblasts of asthmatic patients, with special reference to aspirin-induced asthma. J Allergy Clin Immunol. 2003; 111:1041-8. [PubMed: 12743569]

12. Bochenek G, Nagraba K, Nizankowska E, Szczeklik A. A controlled study of alpha,11beta-PGF 2 (a prostaglandin $\mathrm{D}_{2}$ metabolite) in plasma and urine of patients with bronchial asthma and healthy controls after aspirin challenge. J Allergy Clin Immunol. 2003; 111:743-9. [PubMed: 12704352]

13. Kowalski ML, Ptasinska A, Jedrzejczak M, Bienkiewicz B, Cieslak M, Grzegorczyk J, et al. Aspirin-triggered 15-HETE generation in peripheral blood leukocytes is a specific and sensitive Aspirin-Sensitive Patients Identification Test (ASPITest). Allergy. 2005; 60:1139-45. [PubMed: 16076298]

14. Sanz ML, Gamboa P, de Weck AL. A new combined test with flow cytometric basophil activation and determination of sulfidoleukotrienes is useful for in vitro diagnosis of hypersensitivity to aspirin and other nonsteroidal anti-inflammatory drugs. Int Arch Allergy Immunol. 2005; 136:5872. [PubMed: 15608437]

15. Gamboa P, Sanz ML, Caballero MR, Urrutia I, Antepara I, Esparza R, de Weck AL. The flowcytometric determination of basophil activation induced by aspirin and other non-steroidal anti- 
inflammatory drugs (NSAIDs) is useful for in vitro diagnosis of the NSAID hypersensitivity syndrome. Clin Exp Allergy. 2004; 34:1448-57. [PubMed: 15347380]

16. Boumiza R, Debard AL, Monneret G. The basophil activation test by flow cytometry: recent developments in clinical studies, standardization and emerging perspectives. Clin Mol Allergy. 2005; 30(3):9. [PubMed: 15989690]

17. Kleine-Tebbe J, Erdmann S, Knol EF, MacGlashan DW Jr, Poulsen LK, Gibbs BF. Diagnostic tests based on human basophils: potentials, pitfalls and perspectives. Int Arch Allergy Immunol. 2006; 141:79-90. [PubMed: 16837789]

18. Hemery ML, Arnoux B, Dhivert-Donnadieu H, Rongier M, Barbotte E, Verdier R, Demoly P. Confirmation of the diagnosis of natural rubber latex allergy by the Basotest method. Int Arch Allergy Immunol. 2005; 136:53-7. [PubMed: 15591814]

19. Nettis E, Colanardi MC, Dambra PP, Capuzzimati L, Loria MP, Ferrannini A, Vacca A, Tursi A. Flow cytometric basophil activation test: detection of CD63 expression as a useful aid to diagnosis of latex allergy. Ann Allergy Asthma Immunol. 2006; 97:715-6. [PubMed: 17165288]

20. Sturm GJ, Bohm E, Trummer M, Weiglhofer I, Heinemann A, Aberer W. The CD63 basophil activation test in Hymenoptera venom allergy: a prospective study. Allergy. 2004; 59:1110-7. [PubMed: 15355471]

21. Erdmann SM, Sachs B, Schmidt A, Merk HF, Scheiner O, Moll-Slodowy S, Sauer I, Kwiecien R, Maderegger B, Hoffmann-Sommergruber K. In vitro analysis of birch-pollen-associated food allergy by use of recombinant allergens in the basophil activation test. Int Arch Allergy Immunol. 2005; 136:230-8. [PubMed: 15713985]

22. Sudheer PS, Hall JE, Read GF, Rowbottom AW, Williams PE. Flow cytometric investigation of peri-anaesthetic anaphylaxis using CD63 and CD203c. Anaesthesia. 2005; 60:251-6. [PubMed: 15710010]

23. Ebo DG, Bridts CH, Hagendorens MM, Mertens CH, De Clerck LS, Stevens WJ. Flow-assisted diagnostic management of anaphylaxis from rocuronium bromide. Allergy. 2006; 61:935-9. [PubMed: 16867045]

24. Kvedariene V, Kamey S, Ryckwaert Y, Rongier M, Bousquet J, Demoly P, Arnoux B. Diagnosis of neuromuscular blocking agent hypersensitivity reactions using cytofluorimetric analysis of basophils. Allergy. 2006; 61:311-5. [PubMed: 16436139]

25. Gamboa PM, Sanz ML, Caballero MR, Antepara I, Urrutia I, Jauregui I, Gonzalez G, Dieguez I, De Weck AL. Use of CD63 expression as a marker of in vitro basophil activation and leukotriene determination in metamizol allergic patients. Allergy. 2003; 58:312-7. [PubMed: 12708979]

26. Boumiza R, Monneret G, Forissier MF, Savoye J, Gutowski MC, Powell WS, Bienvenu J. Marked improvement of the basophil activation test by detecting CD203c instead of CD63. Clin Exp Allergy. 2003; 33:259-65. [PubMed: 12580920]

27. Eberlein-Konig B, Varga R, Mempel M, Darsow U, Behrendt H, Ring J. Comparison of basophil activation tests using CD63 or CD203c expression in patients with insect venom allergy. Allergy. 2006; 61:1084-5. [PubMed: 16918511]

28. Yoshimura C, Yamaguchi M, Iikura M, Izumi S, Kudo K, Nagase H, et al. Activation markers of human basophils: CD69 expression is strongly and preferentially induced by IL-3. J Allergy Clin Immunol. 2002; 109:817-23. [PubMed: 11994706]

29. Conesa A, Tassinari P, Rivera H, De Sanctis JB, Bianco N, Aldrey O. Hypodense eosinophils: characterization of surface molecule expression. Allergy Asthma Proc. 2002; 23:117-24. [PubMed: 12001790]

30. Leckie MJ, Jenkins GR, Khan J, Smith SJ, Walker C, Barnes PJ, et al. Sputum T lymphocytes in asthma, COPD and healthy subjects have the phenotype of activated intraepithelial T cells (CD69+ CD103+). Thorax. 2003; 58:23-9. [PubMed: 12511714]

31. National Asthma Education and Prevention Program. Expert Panel Report: Guidelines for the Diagnosis and Management of Asthma Update on Selected Topics--2002. J Allergy Clin Immunol. 2002; 110(5 Suppl):S141-219. [PubMed: 12542074]

32. Schroeder JT, Schleimer RP, Lichtenstein LM, Kreutner W. Inhibition of cytokine generation and mediator release by human basophils treated with desloratadine. Clin Exp Allergy. 2001; 31:136977. [PubMed: 11591186] 
33. Prussin C, Metcalfe DD. Detection of intracytoplasmic cytokine using flow cytometry and directly conjugated anti-cytokine antibodies. J Immunol Methods. 1995; 188:117-28. [PubMed: 8551029]

34. Dahlen B, Boreus LO, Anderson P, Andersson R, Zetterstrom O. Plasma acetylsalicylic acid and salicylic acid levels during aspirin provocation in aspirin-sensitive subjects. Allergy. 1994; 49:439. [PubMed: 8198239]

35. Malbran A, Yeyati E, Rey GL, Galassi N. Diclofenac induces basophil degranulation without increasing CD63 expression in sensitive patients. Clin Exp Immunol. 2007; 147:99-105.

[PubMed: 17177968]

36. Schafer D, Schmid M, Gode UC, Baenkler HW. Dynamics of eicosanoids in peripheral blood cells during bronchial provocation in aspirin-intolerant asthmatics. Eur Respir J. 1999; 13:638-46. [PubMed: 10232440]

37. Lebel B, Messaad D, Kvedariene V, Rongier M, Bousquet J, Demoly P. Cysteinyl-leukotriene release test (CAST) in the diagnosis of immediate drug reactions. Allergy. 2001; 56:688-92. [PubMed: 11421930]

38. Schroeder JT, MacGlashan DW Jr, Lichtenstein LM. Mechanisms and pharmacologic control of basophil-derived IL-4 and IL-13. Int Arch Allergy Immunol. 1999; 118:87-9. [PubMed: 10224347]

39. Gibbs BF. Human basophils as effectors and immunomodulators of allergic inflammation and innate immunity. Clin Exp Med. 2005; 5:43-9. [PubMed: 16096852]

40. Mukai K, Matsuoka K, Taya C, Suzuki H, Yokozeki H, Nishioka K, et al. Basophils play a critical role in the development of IgE-mediated chronic allergic inflammation independently of $\mathrm{T}$ cells and mast cells. Immunity. 2005; 23:191-202. [PubMed: 16111637] 

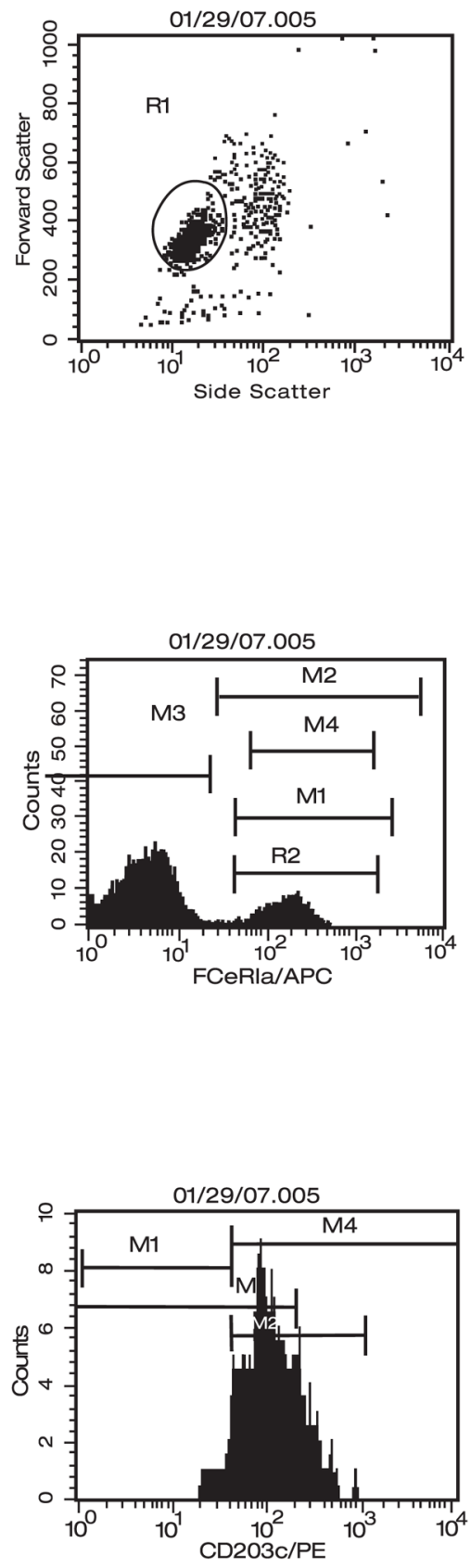

Figure 1.

Primary and secondary gatings for basophils in flow cytometry. 


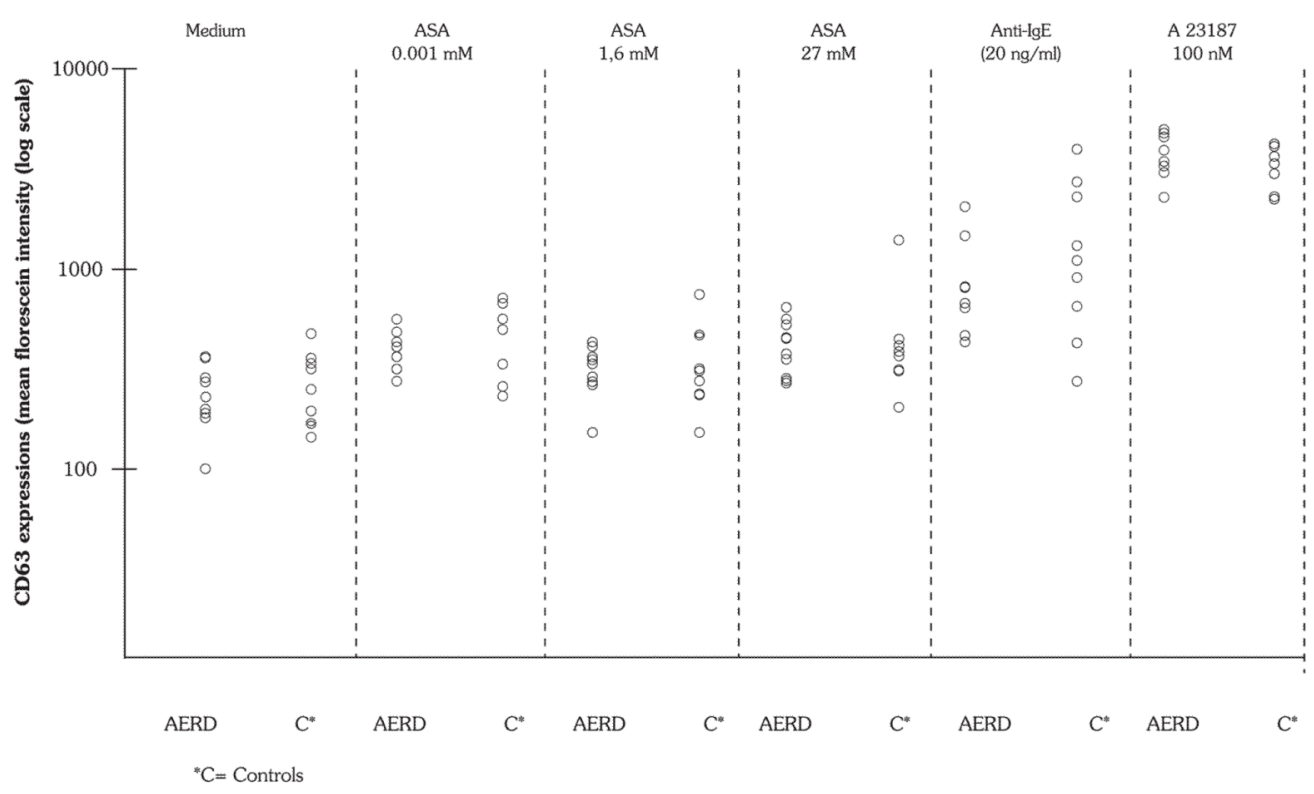

Figure 2.

Baseline and aspirin-stimulated CD63 expression on basophils:. Individual values for the medium alone, three doses of aspirin and two positive controls; ( 


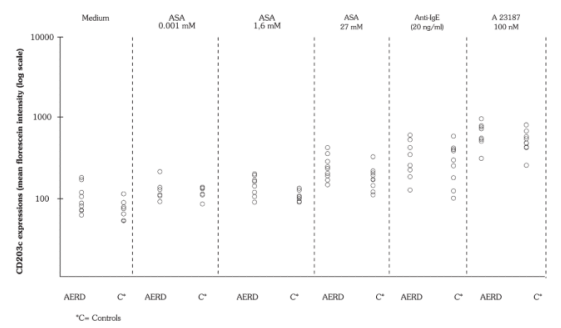

Figure 3.

Baseline and aspirin-stimulated CD203c expression on basophils: Individual values for the medium alone, three doses of aspirin and two positive controls; 


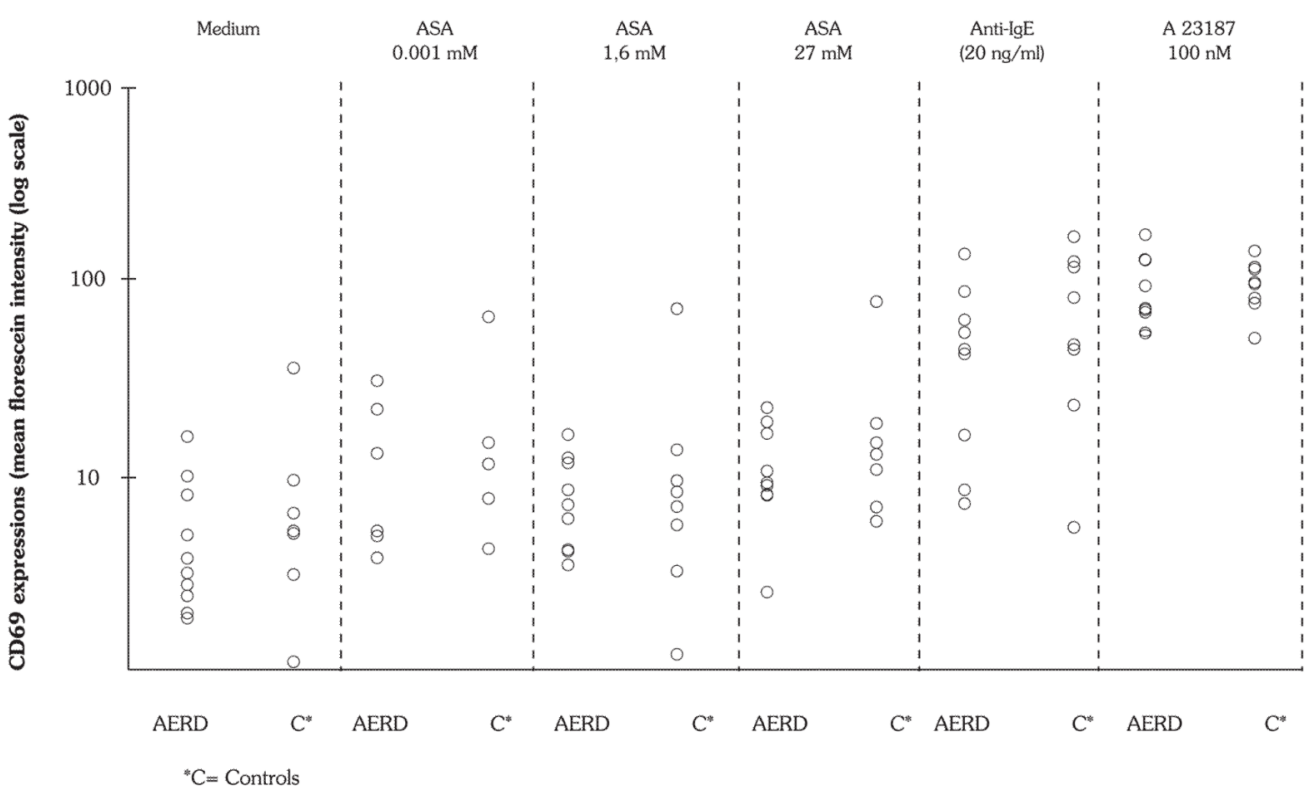

Figure 4.

Baseline and aspirin-stimulated CD69 expression on basophils: Individual values for the medium alone, three doses of aspirin and two positive controls 


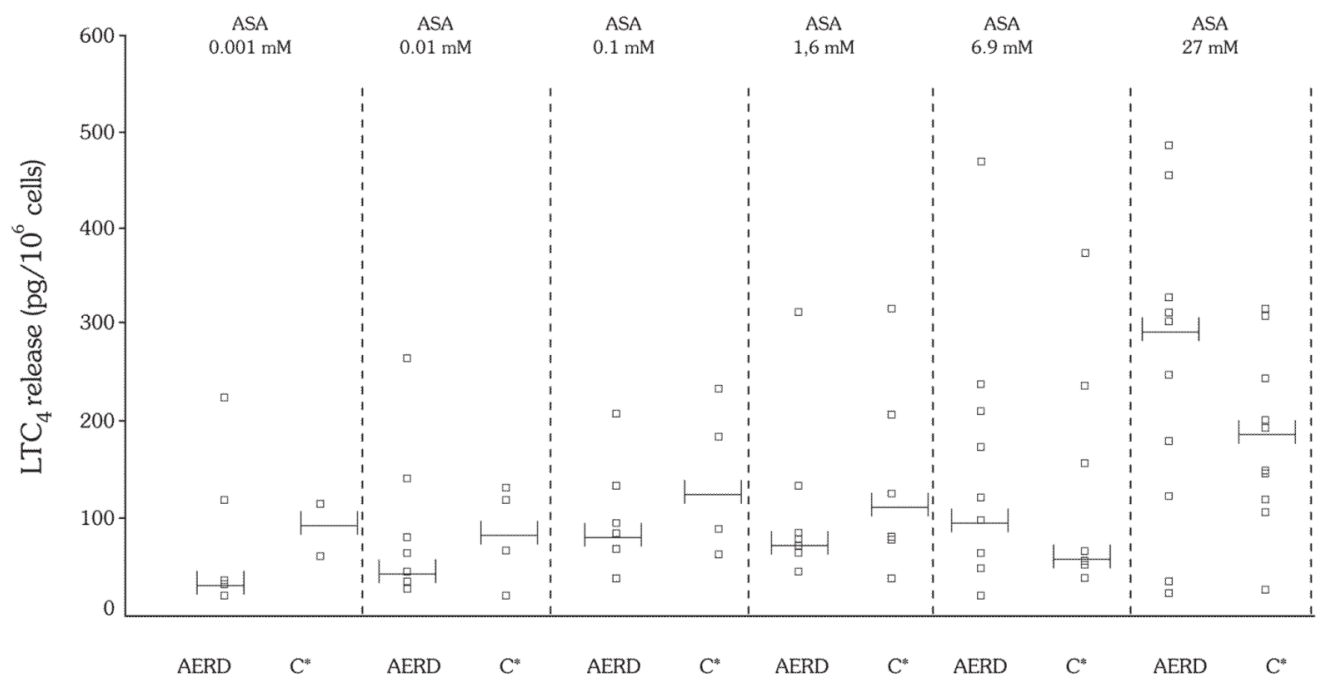

${ }^{*} \mathrm{C}=$ Controls

Figure 5.

Aspirin stimulated $\mathrm{LTC}_{4}$ release from basophil enriched cell suspension. Error bars represent medians. 
Table 1

Demographics and disease characteristics of the patients with AERD

\begin{tabular}{|l|l|}
\hline Variable & \\
\hline Age (mean \pm SEM) years & $45 \pm 3$ \\
\hline Gender (Female/Male) $\mathrm{n}$ & $7 / 3$ \\
\hline Disease duration (mean \pm SEM) years & $13.5 \pm 3$ \\
\hline Duration of aspirin sensitivity (mean \pm SEM) years & $8.1 \pm 1.8$ \\
\hline Presence of nasal polyps/chronic rhinosinusitis $\mathrm{n}(\%)$ & $10(100 \%)$ \\
\hline
\end{tabular}

